\title{
Impact of Age, Tumor Size, Lymph Node Metastasis, Stage, Receptor Status and Menopausal Status on Overall Survival of Breast Cancer Patients in Pakistan
}

\author{
Humera Mahmood ${ }^{1,2 *}$, Mohammad Faheem² ${ }^{2}$ Sana Mahmood ${ }^{2}$, Maryam Sadiq ${ }^{3}$, \\ Javaid Irfan ${ }^{2}$
}

\begin{abstract}
Background: Survival of breast cancer patients depends on a number of factors which are not only prognostic but are also predictive. A number of studies have been carried out worldwide to find out prognostic and predictive significance of different clinicopathological and molecular variables in breast cancer. This study was carried out at Nuclear Medicine, Oncology and Radiotherapy Institute (NORI), Islamabad, to find out the impact of different factors on overall survival of breast cancer patients coming from Northern Pakistan. Materials and Methods: This observational retrospective study was carried out in the Oncology Department of NORI Hospital. A total of 2,666 patients were included. Data were entered into SPSS 20. Multinomial logistic regression analysis was performed to determine associations of different variables with overall survival. $P$ values $<0.05$ were considered significant. Results: The mean age of the patients was 47.6 years, $49.5 \%$ being postmenopausal. Some 1,708 were ER positive and 1,615 were PR positive, while Her 2 neu oncogene positivity was found in 683 . A total of 1,237 presented with skin involvement and 426 had chest wall involvement. Some $1,663 \mathrm{had}>5 \mathrm{~cm}$ tumors. Lymph node involvement was detected in 2,131. Overall survival was less than 5 years in 669 patients, only 324 surviving for more than 10 years, and in the remainder overall survival was in the range of 5-10 years. Conclusions: Tumor size, lymph node metastases, receptor status, her 2 neu positivity, skin involvement, and chest wall involvement have significant effects whereas age and menopausal status have no significant effect on overall survival of breast cancer patients in Pakistan.
\end{abstract}

Keywords: Breast cancer - receptor status - Her 2 Neu oncogene - postmenopausal - overall survival - Pakistan

Asian Pac J Cancer Prev, 16 (3), 1019-1024

\section{Introduction}

Breast cancer is a major public health problem for women throughout the world. Every year more than 1.2 million women are diagnosed with breast cancer. The incidence of breast cancer in Pakistan is high compared to other Asian countries and is approximately 2.5 times that in India and Iran (Asif et al., 2014). It has been found to constitute $33 \%$ of all cancers in females registered at Nuclear Medicine, Oncology and Radiotherapy Institute (NORI) Islamabad (NORI Annual Report 2010-2012). Survival of breast cancer varies widely depending on a number of factors. Generally the major cause of short survival in breast cancer is formation of metastases (Rezaienzadeh et al., 2012).

Although no single special factor has been reported for breast cancer many useful clinicopathological factors have been identified. Treatment of breast cancer can be evaluated with the help of clinically predictive and prognostic factors. An ideal prognostic factor should be capable of predicting the development of metastases and overall survival in all patients and should be measurable following initial treatment (Bekir et al., 2003; Goldhrisch et al., 2005). A number of host and disease related factors including age at diagnosis, menopausal status, tumor size, nuclear grade, surgical margin status, estrogen and progesterone receptors, lymphovascular invasion around tumor matrix and axillary lymph node involvement are well established prognostic factors in breast cancer survival (Goldhrisch et al., 2007). Her $2 \mathrm{Neu}$ gene amplification is another important prognostic and predictive factor for breast cancer. Approximately $20 \%$ of breast cancer patients have Her 2 Neu gene amplification which results in glycoprotein overexpression (Burstein $\mathrm{HJ}$ et al., 2008). This oncogene is associated with tumour aggressiveness and chemoresistanceMaksimovic S (2009). Generally, the frequency of receptor positivity is inversely correlated with Her 2 Neu (Kariya et al., 2005; Kaptain et al., 2001; Almasri and Al Hamad, 2005). ER, PR negativity, and Her $2 \mathrm{Neu}$ positivity have been found to be associated with higher tumour grade, larger tumour size, higher degree of lymph node involvement, and aggressive 
histopathological type (Ayadi et al., 2008). In a study conducted by Abedayo et al (2009) overall survival (OS) and disease free survival (DFS) were found to be $84.6 \%$ and $75.9 \%$ in Her 2 neu positive patients whereas OS was $88.5 \%$ and DFS $84.7 \%$ in Her 2 neu negative patients. Postmenopausal breast cancer women generally do better as compared to premenopausal patients. (Christine et al., 1989)Women younger than 36 years usually present with advanced stage, lymphovascular invasion, higher grade, higher chance of receptor negativity and Her 2 neu positivity and hence poor prognosis(Goksu et al., 2014). Numerous studies done in many countries have shown the value of using tumor size and nodal status to estimate prognosis in breast cancer. Study done by Michaelson et al revealed that both tumor size and the number of positive lymph nodes make independent contribution to the lethality of invasive breast carcinoma. Lethality increased with increase in the number of positive lymph nodes(Michaelson et al., 2003).

Although a number of studies have been done worldwide to estimate survival of breast cancer patients with regard to demographics and clinicopathological features but no such study has been done in Pakistan. Contrary to Europe and America, in Pakistan more than $60 \%$ of breast cancer patients present at an advanced stage of the disease. This means the biological behavior of breast cancer in Pakistani population is different. Cancer patients from Northern Pakistan which include the twin cities of Rawalpindi, Islamabad, upper Punjab, Khyber Pakhtunkhwa, GilgitBaltistan, Kashmir, and Hazara attend NORI. This study was carried out to find out the impact of tumor size, skin and chest wall involvement, lymph node metastases, receptor status, Her 2 neu positivity and menopausal status on survival of breast cancer cohort from Northern Pakistan, registered in Oncology department of NORIHospital, Islamabad.

\section{Materials and Methods}

This observational retrospective study was conducted in the Oncology Department of Nuclear Medicine, Oncology \& Radiotherapy Institute (NORI), Islamabad, after review and approval by the Institutional Ethics Review Committee. All those patients were excluded from the study who were treated for local recurrence, with only a carcinoma in situ, who received neoadjuvant therapy, with primary metastatic disease and with multifocal disease. Data were analyzed for 2666 patients with histopathologically proven diagnosis of invasive ductal carcinoma of the breast. All the patients analyzed were treated in Oncology department of NORI between January 2000 and December 2009. Data were entered into SPSS 20. The status of estrogen receptor (ER), progesterone receptor (PR), and Her 2 Neu oncogene had been sought by immunohistochemistry, and the presence of Her 2 Neu oncogene was confirmed by the Fluorescent In situ Hybridization (FISH) technique in each patient. Age (years), menopausal status, tumour size $(\mathrm{cm})$, and the presence or absence of nodal involvement (including number), skin involvement and chest wall involvement were noted along with ER, PR, and Her 2 Neu status on a specifically designed proforma. Mean \pm standard deviation was calculated for continuous variables like age and tumor size. The frequency and percentage were calculated for categorical variables like skin and chest wall involvement, ER, PR, and Her 2 Neu status, lymph node involvement and menopausal status. Overall survival was estimated for all patients. It was calculated from the date of diagnosis (date of histopathology) to the date of death. After completion of treatment all the patients had been followed 3 monthly during first year, 6 monthly upto 5years and annually thereafter. The association of age, menopausal status, tumor size, skin involvement, chest wall involvement, lymph node involvement, number of nodes involved, ER, PR, and Her 2 Neu status with overall survival was sought employing multinomial logistic regression analysis. Multinomial regression was used because survival was subdivided into 3 categories i.e. $<5$ years, 5-10 years and $>10$ years. $\mathrm{P}$ value $<0.05$ was considered significant. Confidence interval was $95 \%$. Cross tabulation was done to find out percentage of patients for overall survival in relation to different parameters.

\section{Results}

The study comprised of 2666 patients. The mean age \pm standard deviation of the patients studied was $47.57 \pm$ 12.026 years and median age was 45 years. Range was 16 to 100 years. In the study population $49.5 \%$ (1321) women were postmenopausal. Seventeen hundred and eight (64.1\%) patients were ER positive and 1615 (60.6\%) were PR positive. Her 2 neu oncogene was found positive in $683(25.6 \%)$ patients. Twelve hundred and thirty seven (1237) i.e $46.4 \%$ presented with skin involvement while $426(16 \%)$ had chest wall involvement. As far as primary tumor size is concerned $1663(62.4 \%)$ patients had $>5 \mathrm{~cm}$ tumor. Only $101(3.8 \%)$ patients presented with tumor upto $2 \mathrm{~cm}$ in size. Mean tumor size \pm SD was $6.65 \mathrm{~cm} \pm 3.08 \mathrm{~cm}$. Minimum tumor size recorded was $0.8 \mathrm{~cm}$ and maximum was $20 \mathrm{~cm}$. For convenience in analysis age and tumor size were divided into groups. Lymph node involvement was detected in 2131 (79.9\%) patients. More than 4 nodes were found to be involved in 1325 patients while 526 patients had no nodal involvement. Overall survival was less than 5 years in $669(25.1 \%)$ patients and only 324 $(12.2 \%)$ survived for more than 10 years and in rest of the patients overall survival was in the range of 5-10 years.

Multinomial regression analysis of survival when done for different variables separately showed significant association of tumor size, skin involvement, chest wall involvement, receptor status, Her 2 neu oncogene status, lymph node involvement and number of lymph nodes involved with overall survival ( $p$ value $<0.05$ ). However in our study age and menopausal statuswere not found to significantly affect overall survival ( $\mathrm{p}$ value $>0.05$ ). The likelihood ratio chi-square was 2.158 for menopause and 21.739 for age.For tumor size the likelihood ratio chi-square was 42.885 with a $\mathrm{p}$ value $<0.05$ showing significant effect of tumor size in the study. The relative $\log$ odds of survival for less than 5 years versus more than 10 years decreased by 0.0948 and for 5-10 years 
versus more than 10 years decreased by 0.260 moving from the tumor size $>5 \mathrm{~cm}$ to upto $2 \mathrm{~cm}$. This means that overall survival decreased form smaller tumor size to larger tumor size. More patients with tumor size $\leq$ $2 \mathrm{~cm}$ had overall survival more than 10 years.For skin involvement the likelihood ratio chi-square was 249.103 with a p-value $<0.05$ showing that skin involvement had significant effect on overall survival. The relative log odds of survival for less than 5 years versus more than 10 years increased by 0.021 moving from no skin involvement to skin involvement while it decreased by 1.283 for $5-10$ years survival versus $>10$ years survival. The relative risk ratio (RRR) switching from no skin involvement to skin involvement was 1.022 for 5-10 years survival versus more than 10 years survival and it was 0.277 for less than 5 years survival versus more than 10 years survival. In other words majority of patients with skin involvement had overall survival in the range of 5-10 years. For chest wall involvement the likelihood ratio chi-square of 71.720 with a p-value $<0.05$ showed significant effect of chest wall involvement on overall survival. The relative log odds of survival for less than 5 years survival versus more than 10 years increased by 1.297 when moving from no chest wall involvement to chest wall involvement whereas it increased by 0.432 for 5-10 years versus more than 10 years. The relative risk ratio switching from no chest wall involvement to chest wall involvement was 3.660 for 5-10 years survival versus more than 10 years survival and 1.540 for $<5$ years survival. In other words, the expected 5-10 years survival as well as $<5$ years survival as compared to 10 years OS was higher for patients with involved chest wall.

For lymph node involvement the likelihood ratio chi-square was 72.454 with a p-value $<0.05$ showing significant effect in the model.The relative log odds of survival for less than 5 years versus more than 10 years

Table 1. Frequency and Percentage of Different Clinicopathological Features and Overall Survival

\begin{tabular}{|c|c|c|c|}
\hline Parameter & & $\begin{array}{c}\text { Number } \\
\text { of patients }\end{array}$ & $\begin{array}{c}\text { Patients } \\
(\%)\end{array}$ \\
\hline Mean age in $y r s \pm S D$ & $47.57 \pm 12.026$ & & \\
\hline \multirow[t]{2}{*}{ Menopause } & Premenopausal & 1345 & 50.5 \\
\hline & Postmenopausal & 1321 & 49.5 \\
\hline \multirow[t]{3}{*}{ Tumor size in $\mathrm{cm}$} & $\leq 2$ & 101 & 3.8 \\
\hline & $>2-5$ & 902 & 33.8 \\
\hline & $>5$ & 1663 & 62.4 \\
\hline \multirow[t]{2}{*}{ Skin involvement } & Yes & 1237 & 46.4 \\
\hline & No & 1429 & 53.6 \\
\hline \multirow[t]{2}{*}{ Chest wall involvement } & Yes & 426 & 16 \\
\hline & No & 2240 & 84 \\
\hline \multirow[t]{2}{*}{ Lymph node involvement } & Yes & 2131 & 79.9 \\
\hline & No & 535 & 20.1 \\
\hline \multirow[t]{2}{*}{ Estrogen receptor (ER) } & Positive & 1708 & 64.1 \\
\hline & Negative & 958 & 35.9 \\
\hline \multirow[t]{2}{*}{ Progesterone receptor } & Positive & 1615 & 60.6 \\
\hline & Negative & 1051 & 39.4 \\
\hline \multirow[t]{2}{*}{ Her 2 neu } & Positive & 683 & 25.6 \\
\hline & Negative & 1983 & 74.4 \\
\hline \multirow[t]{3}{*}{ Overall survival } & $\leq 5$ years & 669 & 25.1 \\
\hline & $>5-10$ years & 1673 & 62.8 \\
\hline & $>10$ years & 324 & 12.2 \\
\hline
\end{tabular}

increased by 0.547 and for 5-10 years survivall decreased by 0.520 moving from no nodal involvement to nodal involvement. The relative risk ratio switching from no nodal involvement to nodal involvement was 1.729 for 5-10 years survival versus more than 10 years survival and 0.595 for less than 5 years survival versus more than 10 years survival. In other words, the expected 5-10 years survival was higher for patients having positive lymph nodes. More than 10 year survival was lower for patients with involved nodes.Association of number of nodes with OS revealed the likelihood ratio chi-square of 125.983 with a p-value $<0.05$ showing significant effect. It was observed that probability of lymph node involvement increased linearly with tumor size but such linear relationship between tumor size and number of nodes was not detected.

For estrogen receptor the likelihood ratio chi-square of 63.932 with a p-value $<0.05$ revealed that estrogen receptor status had a significant effect in the study. The relative log odds of survival for less than 5 years versus more than 10 years survival increased by 1.109 and for 5-10 years versus more than 10 years increased by 1.126 moving from positive estrogen receptor status to negative one. The relative risk ratio switching frompositive receptor status to negative was 4.214 for 5-10 years survival versus more than 10 years survival 4.189 for less than 5 years survival versus more than 10 years survival. In other words, more patients with positive estrogen receptor status had overall survival of $>10$ years as compared to receptor negative patients. For progesterone receptor the likelihood ratio chi-square of 90.595 with a $p$-value $<0.05$ showed progesterone receptor status had significant effect in the model. The relative log odds of survival for less than 5 years versus more than 10 years increased by 1.087 and for 5-10 years versus more than 10 years increased by 1.331 moving from positive progesterone receptor status to negative status. The relative risk ratio switching from positive progesterone receptor status to negative status was 2.966 for 5-10 years survival versus more than 10 years survival and 3.787 for less than 5 years survival versus more than 10 years survival. In other words, the expected overall survival for more than 10 years was higher for patients with positive progesterone receptor as compared to receptor negative patients.

For lymph node involvement the likelihood ratio chi-square was 72.454 with a p-value $<0.05$ showing significant effect in the model.The relative log odds of survival for less than 5 years versus more than 10 years increased by 0.547 and for 5-10 years survival decreased by 0.520 moving from no nodal involvement to nodal involvement. The relative risk ratio switching from no nodal involvement to nodal involvement was 1.729 for 5-10 years survival versus more than 10 years survival and 0.595 for less than 5 years survival versus more than 10 years survival. In other words, the expected 5-10 years survival was higher for patients having positive lymph nodes. More than 10 year survival was lower for patients with involved nodes.Association of number of nodes with OS revealed the likelihood ratio chi-square of 125.983 with a p-value $<0.05$ showing significant effect. It was observed that probability of lymph node 
Table 2. Impact of Multiple Variables together on Overall Survival

\begin{tabular}{|l|c|l|}
\hline Parameters & $\begin{array}{l}\text { Likelihood ratio } \\
\text { chisquare }\end{array}$ & P value \\
\hline Tumor size with age & 245.619 & $<0.05$ \\
\hline $\begin{array}{l}\text { Tumor size with estrogen recep- } \\
\text { tor status }\end{array}$ & 98.622 & $<0.05$ \\
\hline $\begin{array}{l}\text { Tumor size with progesterone } \\
\text { receptor status }\end{array}$ & 125.293 & $<0.05$ \\
\hline $\begin{array}{l}\text { Tumor size with number of } \\
\text { nodes involved }\end{array}$ & 79.445 & $<0.05$ \\
\hline $\begin{array}{l}\text { Tumor size with Her 2 neu } \\
\text { oncogene status }\end{array}$ & 61.844 & $<0.05$ \\
\hline $\begin{array}{l}\text { Tumor size with both receptors } \\
\text { \& Her 2 neu oncogene status }\end{array}$ & 155.165 & $<0.05$ \\
\hline $\begin{array}{l}\text { Tumor size with both receptors, } \\
\text { Her 2 neu oncogene status \& } \\
\text { number of nodes }\end{array}$ & 255.783 & $<0.05$ \\
\hline $\begin{array}{l}\text { Tumor size with number of } \\
\text { nodes \& age }\end{array}$ & 291.029 & $<0.05$ \\
\hline
\end{tabular}

involvement increased linearly with tumor size but such linear relationship between tumor size and number of nodes was not detected.

For estrogen receptor the likelihood ratio chi-square of 63.932 with a p-value $<0.05$ revealed that estrogen receptor status had a significant effect in the study. The relative log odds of survival for less than 5 years versus more than 10 years survival increased by 1.109 and for 5-10 years versus more than 10 years increased by 1.126 moving from positive estrogen receptor status to negative one. The relative risk ratio switching frompositive receptor status to negative was 4.214 for 5-10 years survival versus more than 10 years survival 4.189 for less than 5 years survival versus more than 10 years survival. In other words, more patients with positive estrogen receptor status had overall survival of $>10$ years as compared to receptor negative patients. For progesterone receptor the likelihood ratio chi-square of 90.595 with a p-value $<0.05$ showed progesterone receptor status had significant effect in the model. The relative log odds of survival for less than 5 years versus more than 10 years increased by 1.087 and for 5-10 years versus more than 10 years increased by 1.331 moving from positive progesterone receptor status to negative status. The relative risk ratio switching from positive progesterone receptor status to negative status was 2.966 for 5-10 years survival versus more than 10 years survival and 3.787 for less than 5 years survival versus more than 10 years survival. In other words, the expected overall survival for more than 10 years was higher for patients with positive progesterone receptor as compared to receptor negative patients.

For Her 2 neu oncogene the likelihood ratio chisquare of 20.990 with a p-value $<0.05$ showed that Her 2 neu oncogene had a significant effect in the model. The relative log odds of survival for less than 5 years versus more than 10 years decreased by 0.452 moving from positive Her 2 neu status to negative status and for 5-10 years versus more than 10 years decreased by 0.667 .
The relative risk ratio switching from positive Her 2 neu status to negative status was 0.636 for 5-10 years survival versus more than 10 years survival and 0.513 for less than 5 years survival versus more than 10 years survival. In other words, the expected overall survival of $>10$ years was higher for patients with negative Her 2 neu oncogene status as compared to Her 2 positive patients. Multinomial regression analysis done to look for impact of two and more than two variables together on overall survival also revealed significant results.

\section{Discussion}

Breast cancer is considered as a heterogeneous condition and so requires evaluation of as many clinical and pathological features as possible to allow for best prediction of survival. The primary objective of this study was to determine the impact of different clinicopathological features on survival of breast cancer. The mean age at diagnosis in our study was 47.5 years which is very similar to that reported in other Asian studies. However, in western population the mean age at diagnosis, as published by Surveillance, Epidemiology and End results (SEER) is 61 years(Hussein $O$ et al., 2013). Majority of our patients presented with nodal involvement, larger tumor size and receptor (ER and PR) positivity. $49.5 \%$ were postmenopausal and $50.5 \%$ were premenopausal in our study population.

The inverse relation between tumor size and survival has been quantitatively known for many years and this is also evident in our study. The likelihood ratio chi-square was 42.885 with a p-value $<0.05$ showing significant effect of tumor size on survival. More patients with tumor size $\leq 2 \mathrm{~cm}$ had overall survival of more than 10 years. A study conducted by Christine et al (1989) comprising of 24,740 patients showed that 5 year survival decreases from $96.3 \%$ to $82.2 \%$ as the diameter of primary tumor increases from less than $2 \mathrm{~cm}$ to $5 \mathrm{~cm}$ or more. Increase in tumor size is also associated with increased risk of nodal involvement. In patients with tumor size of $5 \mathrm{~cm}$ or greater, the probability of nodal involvement is $71.1 \%$. Furthermore, the risk of metastatic disease also increases as the tumor size increases. This was also observed in our study. As the tumor size increased from $<2 \mathrm{~cm}$ to more than $5 \mathrm{~cm}$ the incidence of lymphnode involvement increased from $37 \%$ to $90 \%$. Higher percentage of lymph node involvement was detected in our study. This is because of tumor sizes as large as $20 \mathrm{~cm}$ (range 0.8 to $20 \mathrm{~cm}$ ). Such larger tumor sizes are rarely seen in western countries.

Tumors with signs of skin invasion, such as peau d orange appearance, ulcers, and satellite nodules or chest wall involvement are classified as T4 category in TNM staging system. The 5 year survival decreases to $21 \%$ in patients with T4 tumors (Nair et al., 1993). The prognostic significance of skin only involvement in patients with node negative disease is uncertain. Study conducted by Tada et al showed no significant difference in 10 years disease specific overall survival rates in terms of skin invasion in node negative patients (Tada et al., 2008). Another study conducted showed that in patients with T4b carcinoma, the feature non inflammatory skin involvement has no 
prognostic significance(Guth et al., 2005). Subgroup analysis conducted in our study also showed that greater number of patients with skin only involvement survived for more than 10 years as compared to patients with chest wall involvement.

The involvement of axillary lymphnodes is linked directly to potential for distant recurrence and thus predicts survival. The 5 year survival decreases by $40 \%$ if the lymphnodes get involved (Jafari-Koshki et al., 2014). Moreover the number of nodes involved also predicts survival. One of the indications of adjuvant systemic treatments is presence of lymphnode involvement. Thus lymphnode involvement not only predicts survival but also warrants systemic treatment. In a study conducted the 5-year survival for patients with node-negative disease was $82.8 \%$ compared with $73 \%$ for $1-3$ positive nodes, $45.7 \%$ for $4-12$ positive nodes, and $28.4 \%$ for $>13$ positive nodes(Cianfrocca and Goldstein, 2004). We also observed in our study that $16.5 \%$ patients with less than 5 nodes involved survived for more than 10 years but only $5 \%$ patients with more than 9 nodes involved survived for more than 10 years suggesting decrease in survival with increase in number of nodes involved.

Studies have shown improved outcome in patients with ER, PR positivity and Her 2 neu negativity. This is in accordance with results seen in our study. Patients with ER and PR positivity had statistically significant improved overall survival as compared to ER and PR negative patients $(\mathrm{p}<0.05)$. In the study conducted at NORI, more than 10 year survival was $15 \%$ and $16.5 \%$ in ER positive and PR positive patients respectively but dropped to $5.7 \%$ and $5.3 \%$ in ER negative and PR negative patients respectively. An overall survival of more than 10 years was seen in $13.6 \%$ patients who were Her 2 neu negative and this reduced to almost half i.e. $7.9 \%$ in patients who were Her 2 neu positive. ER and PR positivity is considered not only a prognostic marker but it also predicts response to treatment. Study conducted by Goyanes et al showed that as clinical stage, nuclear grade, histologic grade and lymphnode involvement increased, ER positivity decreased in tumor studied $(\mathrm{p}<0.05)$. Also ER positivity was greater in patients 50 years of age or older (Goyanes et al., 2010). In contrast patients with Her 2 neu positivity are younger, present with higher T stage, nodal involvement and higher grade (Vaz-Luis et al., 2012). Studies have also shown that patients who are ER, PR positive and Her 2 neu negative have decreased incidence of both local and distant recurrence as compared to those who are ER, PR negative and Her 2 neu positive (Shim et al., 2014).

We did not find any association of survival with menopausal status. More than 5 year survival was $73 \%$ in premenopausal women and $76 \%$ in postmenopausal women. A study on breast cancer survival in Hawaii also found no association for menopausal status (Meng et al., 1997).

Although in our study no significant association of overall survival with age was detected. We noticed that none of the patients under 20 years survived for more than 10 years. In the range of $61-70$ years, $25 \%$ of patients had overall survival $>10$ years. For remaining age groups $>10$ year survival was $10-13 \%$.

In addition to stage, lymphnode involvement, number of nodes involved, chest wall involvement, skin involvement, receptor status and Her 2 neu oncogene, other factors may also influence or correlate with prognosis in breast cancer patients. These factors include lymphovascular invasion, histologic subtype, ethnicity, response to treatment, medical co morbidities, positive family history, metastatic disease, recurrent disease and molecular markers like p53 and bcl2. All these factors may not only influence overall survival but also disease free survival. Also treatment factors are very important. The impact of these factors on survival and their prognostic significance should be examined in future studies using larger cohort of patients. The identification of prognostic factors associated with survival may assist physicians in recognizing patients who would benefit from adjuvant treatment.

In conclusion, tumor size, lymph node metastases, receptor status, her 2 neu positivity, skin involvement, and chest wall involvement have significant effect on overall survival of breast cancer patients. Age and menopausal status were not found having significant association with overall survival. All these and other important variables should be considered in determining prognosis and adjuvant treatment in breast cancer patients..

\section{References}

Abedayo A, Onitillo, Jessica M, et al (2009). Breast cancer subtypes based on ER, PR and Her 2 expression : comparison of clinicopathological features and survival. Clinical Medicine and Research, 7, 4-13.

Almasri NM, Al Hamad M (2005). Immunohistochemical evaluation of human epidermal growth factor receptor 2 and estrogen and progesterone receptors in breast carcinoma in Jordan. Breast Cancer Res, 7, 598-604.

Nuclear Medicine, Oncology and Radiotherapy Institute (NORI) (2010-2012) Annual Report. Islamabad, 19.

Asif HM, Sultana S, Akhtar N, et al (2014). Prevalence, risk factors and disease knowledge of breast cancer in Pakistan. Asian Pac J Cancer Prev, 15, 4411-16.

Ayadi L, Khabir A, Amouri H, et al (2008). Correlation of HER2 over-expression with clinicopathological parameters in Tunisian breast carcinoma. World J Surg Oncol, 6, 112-9.

Bekir K, Mithat C, Chamlibel M, et al (2003). Prognostic factors affecting survival and disease free survival in lymph node negative breast carcinoma. J Sur Oncology, 83, 167-72.

Christine L, Carol A, Donald H (1989). Relation of tumor size, lymph node status and survival in 24,740 breast cancer cases. Cancer, 63, 181-7.

Cianfrocca M, Goldstein LJ (2004). Prognostic and predictive factors in early stage breast cancer. The oncologist, 9, 606-16.

Goksu SS, Tastekin D, Arslan D, et al (2014). Clinicopathological features and molecular subtypes of breast cancer in young women (age <35). Asian Pac J Cancer Prev, 15, 6665-8.

Goldhrisch A, Glick JH, Gelber RD, et al (2005). Meeting highlights: International expert consensus on the primary therapy of early breast cancer. Ann Oncol, 16, 1659-583.

Goldhrisch A, Wood WC, Geber RD, et al (2007).Progress and promise: highlights of the international expert consensus on the primary therapy of early breast cancer. Annals of Oncology, 18, 1133-44.

Goyanes RI, Perez XE, Rodriguez RC, et al (2010). Hormone 
receptors and other prognostic factors in breast cancer in Cuba. MEDICC review, 12, 36-40.

Guth U, Wight E, Schotzau A, et al (2005). Breast carcinoma with non inflammatory skin involvement T4b: time to abandon an historic relic from TNM classification. Cancer, 104, 1862-70.

Hussein O, Mosbah M, Farouk O, et al (2013).Hormone receptors and age distibution in breast cancer patients at university hospital in northern Egypt. Breast Cancer: Basic Clin Res, 7, 51-57.

Jafari-Koshki T, Mansourian M, Mokarian F (2014). Exploring factors related to metastasis free survival in breast cancer patients using Bayesian cure models. Asian Pac J Cancer Prev, 15, 9673-78.

Kaptain S, Tan LK, Chen B (2001). Her-2/neu and breast cancer. DiagnMolPathol, 10, 139-52.

Kariya S, Ogawa Y, Nishioka A, et al (2005). Relationship between hormonal receptors, HER-2, p53 protein, bcl-2, and MIB-1 status and the antitumor effects of neoadjuvantanthracycline-based chemotherapy in invasive breast cancer patients. Radiat Med, 23, 189-94.

Maksimovic S (2009). Prognostic value HER-2/neu expression inT1 to T3 breast cancer with axillary lymph node metastasis, Breast Cancer Res, 11 (Suppl 1), 9.

Meng L, Maskarinec G, Wilkens L (1997). Ethnic differences and factors related to breast cancer survival in Hawaii. Int $J$ Epidemiology, 26, 1151-58.

Michaelson JS, Silvesterstein M, Sgroi D, et al (2003). The effect of tumor size and lymph node status on breast carcinoma lethality. Cancer, 98, 2133-43.

Nair MK, Sankaranarayanan R, Nair KS, et al (1993). Overall survival from breast cancer in Kerala, India, in relation to menstrual, clinical and reproductive factors. Cancer, 71, 1791-6.

Rezaienzadeh A, Talei AR, Rejaeefard AR, et al (2012).Vascular invasion as an independent prognostic factor in lymph node negative invasive breast cancer. Asian Pacific J Cancer Prev, 13, 5767-72.

Shim HJ, Kim SH, Kang BJ, et al (2014). Breast cancer recurrence according to molecular subtype. Asian Pac J Cancer Prev, 15, 5539-44.

Tada K, Morizono H, Iijima K, et al (2008). Skin invasion and prognosis in node negative breast cancer: a retrospective study. World J Surg Oncol, 6.

Vaz-Luis I, Ottessen RA, Hughes ME, et al (2012). Impact of hormone receptor status on breast cancer recurrence and clinical outcomes among patients with human epidermal growth factor 2 positive breast cancer in the National comprehensive cancer network. Breast Cancer Res, 14, R129. 